\title{
A TOPOLOGY FOR CERTAIN MEASURE SPACES $\left({ }^{1}\right)$
}

BY

\author{
N. F. G. MARTIN
}

1. Introduction. Henry Blumberg $[2 ; 3]$ has introduced the concepts of a point $x$ of Euclidean space being inexhaustibly approached by a set $S$ and the point $x$ being non-null approached by the set $S$. The first type of approach was generalized by Cargal [4], and in [14] the author placed this generalized approach property into a topological setting so that a point was approached by a set if and only if the point was a limit point of the set in an appropriate topology. The second type of approach, i.e., non-null approach, uses the concept of metric density. In this paper we consider this second type of approach in a totally finite measure space with a distinguished family of measurable sets in terms of which we define metric density. Using the metric density we define a topology $\left({ }^{2}\right)$ in which a point is non-null approached by a set iff the point is a limit point of the set in the defined topology. Various properties of this topology are considered and certain measure theoretic concepts are translated into topological concepts.

We show also that the topological space defined is a measurable space in the sense of Oxtoby [16] if and only if a density theorem holds for the metric density defined.

In the last section we consider the real line with ordinary metric density and restate some theorems of Denjoy and others on derivative functions in terms of this topology and the topology defined in [14].

2. Preliminaries. Most of the statements in this section are standard measure theoretic results but are included here for completeness. Throughout we assume that $(X, \mathscr{S}, m)$ is a totally finite measure space in which $m(X)=1$, and $m$ is complete.

If $E$ is any subset of $X$ we define the outer measure, $m^{*}(E)$, of $E$ by the equation

$$
m^{*}(E)=\inf \{m(F): E \subset F \in \mathscr{S}\} .
$$

2.1. LEMma. If $F$ is any set, there exists a measurable set $E$ such that $E \supset F$ and $m(E)=m^{*}(F)$. Such a set $E$ is called a measurable cover for $F$.

Presented to the Society, January 26, 1961 under the title On derivative functions and May 3, 1963; received by the editors July 18, 1962 and, in revised form, March 8, 1963.

(1) Research supported in part by National Science Foundation grant (NSF-G18853).

(2) This same topology was defined by Goffman, et al. [10; 11] for Euclidean spaces independently but concurrently with the author. See Notices Amer. Math. Soc. 7 (1960), 933. 
Proof. Take $E=\bigcap_{n} E_{n}$, where $E_{n} \supset F$ and $m\left(E_{n}\right) \leqq m^{*}(F)+1 / n$.

2.2. LEMMA. If $G$ is any measurable set and $F$ any set then

$$
m^{*}(F)=m^{*}(G \cap F)+m^{*}(F-G) .
$$

Proof. Let $H$ be a measurable cover for $F$. Then since $H \supset F, H \cap G \supset F \cap G$ and $H-G \supset F-G$ and we have

$$
m^{*}(F)=m(H)=m(H \cap G)+m(H-G) \geqq m^{*}(F \cap G)+m^{*}(F-G) .
$$

2.3. Lemma. If $F$ is any set such that $m^{*}(F)+m^{*}(X-F)=1$ then for any measurable set $G \supset F$,

$$
m(G)=m^{*}(F)+m^{*}(G-F) .
$$

Proof. Since $G$ is measurable, $X-G$ is measurable and

$$
m^{*}(X-F)=m(X-G)+m^{*}(G-F) .
$$

Then

$$
1=m^{*}(F)+m^{*}(X-F)=m^{*}(F)+m(X-G)+m^{*}(G-F)
$$

and since $m(X-G)=1-m(G)$ we have the required result.

2.4. TheOREM. A necessary and sufficient condition that a set $A$ be measurable is that

$$
m^{*}(A)+m^{*}(X-A)=1 .
$$

Proof. The necessity is clear. To prove the sufficiency assume that $A$ is any set satisfying the equation. Let $G$ be a measurable cover for $A$. Then

$$
m(G)=m^{*}(A)+m^{*}(G-A)
$$

and since $m(G)=m^{*}(A)$, we have $m^{*}(G-A)=0$. If $H$ is a measurable cover for $G-A$, then $G-A \subset H$ and $m(H)=0$. Since $m$ is a complete measure, $G-A$ is measurable and if $N=G-A, A=G-N$ and $A$ is measurable.

2.5. Corollary. If $F \subset G \in \mathscr{S}$, then a necessary and sufficient condition that $F \in \mathscr{S}$ is that

$$
m(G)=m^{*}(F)+m^{*}(G-F) .
$$

Proof. The necessity is obvious. To obtain the sufficiency suppose $F$ to be a set satisfying the condition. Since $G \supset F$, and $G$ is measurable, $X-G$ is measurable and by 2.2 we have

$$
m^{*}(X-F)=m(X-G)+m^{*}(G-F) .
$$

Adding this equality to the hypothesized equality gives 


$$
m^{*}(F)+m^{*}(X-F)=m(G)+m(X-G)=m(X)=1 .
$$

2.6. Corollary. If $F$ is a nonmeasurable set and $E$ is a measurable cover for $F$ then $m^{*}(E-F)>0$.

3. Metric density. We now assume that $\mathscr{K}$ is a collection of sequences $\left\{K_{n}\right\}$ of sets from $\mathscr{S}$ such that for each $x \in X$ there exists at least one sequence $\left\{K_{n}\right\} \in \mathscr{K}$ such that

(i) $x \in K_{n}$ for each $n$,

(ii) $m\left(K_{n}\right) \rightarrow 0$ as $n \rightarrow \infty$.

Any sequence of sets satisfying conditions (i) and (ii) we will say converges to $x$. Let $\mathscr{K}(x)$ denote that collection of sequences in $\mathscr{K}$ which converge to $x$.

Let $E$ be any subset of $X$ and $x$ any point in $X$. The upper outer density of $E$ at $x$, denoted by $D^{-*}(E, x)$ and the lower outer density of $E$ at $x$, denoted by $D_{-}^{*}(E, x)$ are defined by the equations

$$
\begin{aligned}
D^{-*}(E, x) & =\sup \left\{\limsup \sup _{n} m^{*}\left(E \cap K_{n}\right) / m\left(K_{n}\right):\left\{K_{n}\right\} \in \mathscr{K}(x)\right\} \\
& =\limsup _{K \rightarrow x} m^{*}(E \cap K) / m(K)
\end{aligned}
$$

and

$$
\begin{aligned}
D_{-}^{*}(E, x) & =\inf \left\{\lim \inf _{n} m^{*}\left(E \cap K_{n}\right) / m\left(K_{n}\right):\left\{K_{n}\right\} \in \mathscr{K}(x)\right\} \\
& =\liminf _{K \rightarrow x} m^{*}(E \cap K) / m(K) .
\end{aligned}
$$

In these equations, if $m\left(K_{n}\right)=0$, we define $m^{*}\left(E \cap K_{n}\right) / m\left(K_{n}\right)$ to be zero.

\subsection{EXAMPLES.}

A. If $X$ is Euclidean $n$-space and $m$ is Lebesgue measure then by defining $\mathscr{K}(x)$ to be the family of all regular sequences of closed sets converging to $x$ (i.e., which contain $x$ and whose diameters shrink to zero) we obtain the regular densities. In case we define $\mathscr{K}(x)$ to be the family of all regular sequences of intervals converging to $x$ or the family of all sequences of intervals converging to $x$, we get respectively the ordinary and strong densities. If we take $\mathscr{K}(x)$ to be the family of all sequences of intervals which converge to $x$ and whose mid-points are $x$, we get symmetric densities. In each case we take $\mathscr{K}$ to be the family consisting of all sequences contained in some $\mathscr{K}(x)$.

B. Let $X$ be a metric space and let $\mathscr{D}$ be an indefinitely fine system on $X$ as defined by Hahn and Rosenthal [12, p. 247]. Then, using the notation of Hahn and Rosenthal, let $\mathscr{K}(x)$ be the family of all sequences of sets from $\mathscr{D}_{x}$ which converge to $x$. If $m$ is a Borel measure on $X$ with no atoms we have densities with respect to indefinitely fine systems.

It is clear that $D^{-*}(E, x) \geqq D_{-} *(E, x)$. In case equality holds we say the outer 
density of $E$ exists at $x$ and denote it by $D^{*}(E, x)$. In case $E$ is measurable, we leave off the word outer and speak of the upper and lower density of $E$ at $x$ and denote these by $D^{-}(E, x)$ and $D_{-}(E, x)$. If $D^{-}(E, x)=D_{-}(E, x)$ we say that the density of $E$ exists at $x$ and denote the common value of $D^{-}$and $D_{-}$by $D(E, x)$. As is commonly done, we call a point at which a set $E$ has (outer) density one a (outer) density point of $E$ and a point at which $E$ has (outer) density zero a (outer) dispersion point for $E$.

3.2. Lemma. For each $x \in X$, the set function $D^{-*}(\cdot, x)$ defined on all subsets of $X$ to the unit interval is monotone nondecreasing and finitely subadditive.

Proof. Since for any two subsets $E$ and $F$ of $X$ and any element $\left\{K_{n}\right\}$ from $\mathscr{K}(x)$, we have

$$
m^{*}\left[(E \cup F) \cap K_{n}\right] / m\left(K_{n}\right) \leqq m^{*}\left(E \cap K_{n}\right) / m\left(K_{n}\right)+m^{*}\left(F \cap K_{n}\right) / m\left(K_{n}\right),
$$

it follows that

$$
\begin{aligned}
\limsup _{K \rightarrow x} m^{*}[(E \cup F) \cap K] / m(K) \leqq & \limsup _{K \rightarrow x} m^{*}(E \cap K) / m(K) \\
& +\limsup _{K \rightarrow x} m^{*}(F \cap K) / m(K)
\end{aligned}
$$

and $D^{-*}(\cdot, x)$ is subadditive.

The monotoneity follows in a similar manner from the monotoneity of $m$

3.3. Lemma. If $D(E, x)$ and $D(F, x)$ exist and $F \cap E=\varnothing$, then $D(E \cup F, x)$ exists and

$$
D(E \cup F, x)=D(E, x)+D(F, x) .
$$

Proof. Since $E$ and $F$ are disjoint, for any $\left\{K_{n}\right\} \in \mathscr{K}(x)$, we have

$$
m\left((E \cup F) \cap K_{n}\right) / m\left(K_{n}\right)=m\left(E \cap K_{n}\right) / m\left(K_{n}\right)+m\left(F \cap K_{n}\right) / m\left(K_{n}\right)
$$

and hence

$$
\begin{aligned}
D \_(E \cup F, x) & =\liminf _{K \rightarrow x} m((E \cup F) \cap K) / m(K) \\
& \geqq \liminf _{K \rightarrow x} m(E \cap K) / m(K)+\liminf _{K \rightarrow x} m(F \cap K) / m(K) \\
& =D(E, x)+D(F, x) \\
& \geqq D^{-}(E \cup F, x) .
\end{aligned}
$$

3.4. Lemma. If $D(F, x)$ and $D(E, x)$ exist and $F \supset E$, then $D(F-E, x)$ exists and

$$
D(F-E, x)=D(F, x)-D(E, x)
$$


Proof. Since $F \supset E$ and each set is measurable we have for any $\left\{K_{n}\right\} \in \mathscr{K}(x)$,

$$
m\left((F-E) \cap K_{n}\right) / m\left(K_{n}\right)=m\left(F \cap K_{n}\right) / m\left(K_{n}\right)-m\left(E \cap K_{n}\right) / m\left(K_{n}\right)
$$

and therefore

$$
\begin{aligned}
D \_(F-E, x) & =\liminf _{K \rightarrow x} m((F-E) \cap K) / m(K) \\
& \geqq \liminf _{K \rightarrow x} m(F \cap K) / m(K)-\limsup _{K \rightarrow x} m(E \cap K) / m(K) \\
& =D(F, x)-D(E, x) \\
& =\limsup _{K \rightarrow x} m(F \cap K) / m(K)-\liminf _{K \rightarrow x} m(E \cap K) / m(K) \\
& \geqq D^{-}(F-E, x) .
\end{aligned}
$$

3.5. ThEOREM. A necessary and sufficient condition that a measurable set $E$ have density at $x$ is that

$$
D^{-}(E, x)+D^{-}(X-E, x)=1 .
$$

Proof. The necessity follows immediately from Lemmas 3.3 and 3.4. To obtain the sufficiency note that, since $E$ is measurable,

$$
m\left(E \cap K_{n}\right) / m\left(K_{n}\right)+m\left((X-E) \cap K_{n}\right) / m\left(K_{n}\right)=1
$$

for any $\left\{K_{n}\right\} \in \mathscr{K}(x)$. Therefore

$$
\begin{aligned}
D^{-}(E, x)+D^{-}(X-E, x) & =1 \\
& =\liminf _{K \rightarrow x}[m(E \cap K) / m(K)+m((X-E) \cap K) / m(K)] \\
& \leqq D_{-}(E, x)+D^{-}(X-E, x),
\end{aligned}
$$

and we have $D^{-}(E, x) \leqq D_{-}(E, x)$.

3.6. LEMma. If $A$ is any set and $E$ is a measurable cover for $A$, then for any $x \in X, D^{-*}(A, x)=D^{-}(E, x)$.

Proof. We show that if $K$ is any measurable set then $E \cap K$ is a measurable cover for $K \cap A$. This follows since

$$
\begin{aligned}
m(E \cap K)+m(E-K) & =m(E)=m^{*}(A) \\
& \leqq m^{*}(A \cap K)+m^{*}(A-K) \\
& \leqq m^{*}(A \cap K)+m(E-K) .
\end{aligned}
$$

4. The density topology. Let $\mathscr{U}$ be the family of subsets of $X$ defined by

$$
\mathscr{U}=\left\{U: D^{-*}(X-U, x)=0 \text { for all } x \text { in } U\right\} .
$$


4.1. THEOREM. The family $\mathscr{U}$ of sets forms a topology for $X$.

Proof. It is clear that $\varnothing$ and $X$ are members of $\mathscr{U}$. Suppose $U_{1}$ and $U_{2}$ are members of $\mathscr{U}$. Then if $U_{1} \cap U_{2} \neq \varnothing$, for any $x \in U_{1} \cup U_{2}$, we have

$$
\begin{aligned}
D^{-*}\left(X-U_{1} \cap U_{2}, x\right) & =D^{-*}\left(\left(X-U_{1}\right) \cup\left(X-U_{2}\right), x\right) \\
& \leqq D^{-*}\left(X-U_{1}, x\right)+D^{-*}\left(X-U_{2}, x\right) \\
& =0
\end{aligned}
$$

and hence $U_{1} \cap U_{2} \in \mathscr{U}$. Next let $\left\{U_{\alpha}\right\}$ be any family of sets from $\mathscr{U}$ and denote their union by $U$. If $x$ is some member of $U$, then there is some $\alpha$ such that $x$ is in $U_{\alpha}$ and since $X-U_{\alpha} \supset X-U$ we have

$$
D^{-*}(X-U, x) \leqq D^{-*}\left(X-U_{\alpha}, x\right)=0 .
$$

The topology $\mathscr{U}$ will be called the density topology for $X$.

It is not clear yet whether the open sets in the density topology are measurable or not, and indeed they may not be. However, if we further restrict our family $\mathscr{K}$ we get a condition for measurability which insures the measurability of the sets in $\mathscr{U}$, i.e., the open sets.

We will say a "weak density theorem holds for $\mathscr{K}$ " if for every measurable set $E$ almost every point of $E$ is a density point for $E$, and a "density theorem holds for $\mathscr{K}$ " if for every set $A$ almost every point of $A$ is an outer density point for $A$. The existence of a density theorem for a family $\mathscr{K}$ is closely related to the existence of a Vitali theorem for the family of sets which make up the sequences in $\mathscr{K}$. The study of various properties of this family which lead to a Vitali theorem and density theorem may be found in Hahn and Rosenthal [12], Denjoy [9], Trjitzinsky [19], and Comfort and Gordon [6].

4.2. THEOREM. A weak density theorem holds if and only if a density theorem holds.

Proof. It is clear that a weak density theorem holds if a density theorem holds. To prove the converse suppose $A$ is any subset of $X$ and let $M$ be a measurable cover for $A$. Almost all points of $M$ are density points of $M$ and hence by Lemma 3.6, outer density points of $A$. But $M \supset A$, so almost all points of $A$ are outer density points of $A$.

4.3. THEOREM. If a density theorem holds a necessary and sufficient condition that a set $E$ be measurable is that almost all points in the complement of $E$ are outer dispersion points for $E$.

Proof. Suppose that $A$ is nonmeasurable. We will show that the set of outer density points of $A$ which are not in $A$ has positive outer measure. Let $E$ be a measurable cover for $A$. By the density theorem hypothesis almost all points of $E$ 
are density points of $E$, hence almost all points of $E-A$ are density points of $E$. But since $E$ is a measurable cover for $A$, every density point of $E$ is an outer density point of $A$, hence almost all points of $E-A$ are outer density points of $A$. Since $A$ is nonmeasurable $m^{*}(E-A)>0$. The necessity follows immediately from Theorem 3.5 .

4.4. Corollary. If a density theorem holds, the open sets are measurable.

Proof. If $U \in \mathscr{U}$, every point of $X-(X-U)$ is an outer dispersion point of $X-U$ by definition. Hence $X-U$ and $U$ are measurable.

4.5. THEOREM. If a density theorem holds and if $U$ is open, every point of $U$ is a density point of $U$.

Proof. This theorem follows from Corollary 4.4 and Theorem 3.5 and the definition of the family $\mathscr{U}$.

For Euclidean space, Goffman et al. $[10 ; 11]$ defined a density topology by designating those sets all of whose points are density points to be open sets. Thus Goffman requires a priori that the open sets in his topology are measurable. We will say a set $E$ is $G$-open if it is measurable and every point in $E$ is a density point of $E$.

4.6. TheOREM. Every G-open set is open and if a density theorem holds for $\mathscr{K}$ then every open set is G-open.

Proof. This follows immediately from Theorems 4.5, 3.5 and the definition of open sets.

Thus, we see that the $G$-open sets coincide with open sets if a density theorem holds. In case $X$ is as defined in example $3.1 \mathrm{~A}$, our topology coincides with Goffman's topology.

It is not true, even under the assumption of a density theorem, that every point of a set being an outer density point for the set implies that the set is open. Indeed, Blumberg [1] has proven a theorem for Euclidean spaces which is also true in our context provided a density theorem holds for $\mathscr{K}$.

4.7. TheOREm (Blumberg). If a density theorem holds for $\mathscr{K}$ and if $E$ is any nonmeasurable set then there exist two disjoint sets $M$ and $P$ such that $E=M \cup P, M$ is measurable, $P$ is nonmeasurable and for every $x \in P$,

$$
D^{*}(P, x)=D^{*}(X-P, x)=1 .
$$

Proof. By the method of exhaustion determine a set $M$ such that $M$ is a measurable subset of $E$ for which $m(M)=\sup \{m(H): E \supset H \in \mathscr{S}\}$. Define $N=E-M$. If $C$ is a measurable cover of $N$, then $C$ is also a measurable cover of $C-N$, since otherwise the interior measure of $N$ would be positive, and $N$ would contain measurable sets of positive measure. By the density theorem, almost all points 
of $N$ are outer density points of $N$. Let $P$ be the set which consists of those points of $N$ which are outer density points of $N$. Then $m^{*}(N)=m^{*}(P)$ and $m^{*}(X-N)$ $=m^{*}(X-P)$. If $x \in P$, then $D^{*}(P, x)=1$ and if $C$ is a measurable cover for $P$, $D(C, x)=1$. But $C$ is also a measurable cover for $X-P$ and hence $D^{*}(X-P, x)$ $=D(C, x)=1$.

It is an easy consequence of this theorem that every point of a set being an outer density point for the set does not imply that the set is open.

Throughout the remainder of the paper we shall assume that a density theorem holds for the family $\mathscr{K}$.

4.8. Lemma. If $E$ is measurable then the interior of $E$ is the set of density points of $E$ which are contained in $E$.

Proof. Let $H=\{x: x \in E, D(E, x)=1\}$. By the density theorem $E-H=N$ where $m(N)=0$ so $H=E-N$ is measurable and $m(H)=m(E)$. Furthermore $D(E, x)=D(H, x)$ for every $x$ for which either exists. Therefore for every $x \in H, D(H, x)=1$ so $H$ is open by Theorem 4.6 and hence $H \subset E^{\circ}$, the interior of $E$. Let $x \in E^{\circ}$. Since $E^{\circ}$ is open $D\left(E^{\circ}, x\right)=1$ and since $E^{\circ} \subset E, D\left(E^{\circ}, x\right) \leqq D_{-}(E, x)$ and hence $D(E, x)=1$. Thus $x \in H$.

4.9. THEOREM. A necessary and sufficient condition that a point $x$ be a limit point for the set $E$ is that $D^{-*}(E, x)>0$.

Proof. Suppose $D^{*}(E, x)=0$. If $H$ is a measurable cover for $E, D(H, x)=0$ and if $x$ is not a limit point for $H$, then $x$ is not a limit point for $E$, since $H \supset E$. We show that $x$ is not a limit point of $H$. Since $H$ is measurable, $D(X-H, x)=1$. Thus the set

$$
N=(X-H)^{\circ} \cup\{x\}=\{z: D(X-H, z)=1, z \in X-H\} \cup\{x\}
$$

is a neighborhood of $x$ such that $N \cap E-\{x\}=\varnothing$.

Next suppose $x$ is not a limit point of $E$. Then there exists a neighborhood $U$ of $x$ such that $U \cap E-\{x\}=\varnothing$, so that $E \subset X-(U-\{x\})=(X-U) \cup\{x\}$. Since $U$ is open, and $x \in U, D(X-U, x)=0$ and

$$
D^{-*}(E, x) \leqq D(X-U, x)=0 .
$$

4.10. Corollary. A necessary and sufficient condition that a set E have measure zero is that it be closed and discrete in the density topology.

Proof. In case $m(E)=0$, then $D(E, x)=0$ for every $x$ and by Theorem $4.9, E$ has no limit points, hence is closed and discrete. In case $E$ is closed and discrete it has no limit points and by Theorem $4.9, D^{*}(E, x)=0$ for all $x \in X$. But, by the density theorem $D^{*}(E, x)=1$ for almost all $x \in E$, hence $m(E)=0$.

4.11. THEOREM. A necessary and sufficient condition that a set be nowhere dense in the density topology is that it have measure zero. 
Proof. In case $m(E)=0, E$ is closed and discrete and therefore nowhere dense. Since every nowhere dense set is a subset of a closed nowhere dense set, all we need consider for the necessity is a closed nowhere dense set $E$. Since $E$ is closed, it is measurable and by Lemma $4.8, E^{\circ}=\{x: x \in E, D(E, x)=1\}$. Since $E^{\circ}=\varnothing, E$ contains no density points and $m(E)=0$ by the density theorem.

4.12. Corollary. A necessary and sufficient condition that a set be first category is that it have measure zero.

Proof. Follows immediately from 4.11, since $m$ is $\sigma$-additive.

4.13. CoRollary. The topological space $(X, \mathscr{U})$ is a Baire space, i.e., every nonempty open set is second category.

Proof. If $U$ is a nonempty open set, there are points in $U$ and for each such point $x, D(U, x)=1$. Hence $U$ has a limit point and by Corollary $4.10, m(U)>0$. Thus by Corollary $4.12, U$ is not first category.

5. Category measure. J. C. Oxtoby [16] has defined measures for topological spaces which he calls category measures. A measure $\mu$ on a topological space is a category measure if the $\mu$-null sets are the first category sets and the family of $\mu$-measurable sets is the family of sets satisfying the property of Baire, i.e., $E$ is $\mu$-measurable if $E$ may be written as the symmetric difference of an open set and a first category set.

5.1. THEOREM. The measure $m$ is a category measure for $(X, \mathscr{U})$.

Proof. The first condition on the sets of measure zero is just the statement of Corollary 4.12. Let $E$ be a measurable set. Since $E$ is measurable, $E=E^{\circ} \cup P$, where $P$ is the set of points in $E$ which are not density points of $E$, and $m(P)=0$. Thus $E=E^{\circ} \cup P=E^{\circ} \triangle P$ and $E$ satisfies the condition of Baire. If $E$ satisfies the Baire condition $E=U \triangle P$ wher $U \in \mathscr{U}$ and $P$ is first category, by Corollaries 4.4 and $4.12, E$ is measurable.

In his paper Oxtoby showed that if a topological space is a measurable space, i.e., has a category measure defined for it, and is a Baire space then the algebra of regular open sets (sets which are interiors of closed sets) is isomorphic to the measure algebra. Since our space $(X, \mathscr{U})$ is a Baire space with a category measure, the algebra of regular open sets is isomorphic to the measure algebra.

5.2. THEOREM. A necessary and sufficient condition that a set $G$ be a regular open set in the density topology is that $x$ is a density point of $G$ if and only if $x$ is a member of $G$.

Proof. Suppose first that $x \in G$ if and only if $D(G, x)=1$. $G$ is open by Theorem 4.6 and if

$$
P=\left\{x: x \notin G, D_{-}(G, x)>0\right\}
$$


then $G^{-}=G \cup P$ and $m(P)=0$. Since $P \cap G=\varnothing, G^{-}=G \triangle P$ and for $x \in X$, $D\left(G^{-}, x\right)=D(G, x)$. Since $G^{-}$is measurable

$$
\begin{aligned}
G^{-\circ} & =\left\{x: x \in G^{-}, D\left(G^{-}, x\right)=1\right\} \\
& =\left\{x: x \in G^{-}, D(G, x)=1\right\} .
\end{aligned}
$$

But by hypothesis, $D(G, x)=1$ implies $x \in G$. Therefore $G^{-\circ} \subset G$. Since $G^{-} \supset G$ and $G$ is open, $G^{-\circ} \supset G$ and $G$ is a regular open set.

Next suppose that $G$ is a regular open set. Since $G$ is open, $D(G, x)=1$ for every $x \in G$. Suppose there exists a point $x_{0} \notin G$ such that $D\left(G, x_{0}\right)=1$. Then as before $G^{-}=G \cup P$ and $D(G, x)=D\left(G^{-}, x\right)$ so that

$$
G^{-\circ}=\left\{x: x \in G^{-}, D(G, x)=1\right\} .
$$

Since $x_{0} \in P, x_{0} \in G^{-\circ}$ but $x_{0} \notin G$. Thus $G \neq G^{-\circ}$ and $G$ is not regular open, contrary to assumption. Thus $D(G, x)=1$ implies that $x$ is a member of $G$.

From Oxtoby's results and Theorem 5.2 it follows that the measure algebra of $m$, i.e., the Boolean algebra of $m$-measurable sets mod the ideal of sets of measure zero, is isomorphic to the family $\mathscr{R}$ of all sets $G$ satisfying the conditicn $x \in G$ if and only if $D(G, x)=1$, where the Boolean operations in $\mathscr{R}$ are defined by

$$
\begin{aligned}
G_{1} \vee G_{2} & =\left\{x: D\left(G_{1} \cup G_{2}, x\right)=1\right\}, \\
G_{1} \wedge G_{2} & =G_{1} \cap G_{2}, \\
-G & =\{x: D(X-G, x)=1\} .
\end{aligned}
$$

In the proof of Theorem 5.1, we used very strongly the fact that a density theorem was satisfied by the family $\mathscr{K}$. As a matter of fact we show next that a density theorem is necessary for $m$ to be a category measure.

5.3. THEOREM. If $(X, \mathscr{S}, m)$ is a measure space with $m(X)=1$ and $\mathscr{K}$ is a family of sequences of measurable sets satisfying the conditions in $\$ 3$, then $m$ is a category measure for the density topology on $X$ only if a density theorem holds for $\not K$.

Proof. From Theorem 4.2 we need only consider measurable sets $E$. Since $m$ is assumed to be a category measure for the density topology, $E=U \triangle P$ where $U$ is open and $P$ first category and hence $m(P)=0$. Thus $m(U \triangle E)=0$ and for any $x, D^{-}(U, x)=D^{-}(E, x)$, and since $U$ is open, $D(U, x)=1$ for every $x \in U$. Since $U-P \subset E$, if $H=\{x: x \in E, D(E, x)=1\}$, we have $U-P \subset H \subset E$. Since $m(U-P)=m(U)=m(E), H$ is measurable and $m(H)=m(E)$.

6. Measurable and continuous functions. In this section we investigate the relationship between the functions which are continuous in the density topology and measurable functions. All the functions considered in this section are assumed to be either real valued or extended real valued. The usual result is Lusin's theorem; for the density topology we have the following result. 
6.1. THEOREM. In the density topology the following statements are equivalent

A. Lusin's theorem.

B. $m$ is a regular Borel measure.

C. The density topology is quasi-regular, i.e., every nonempty open set contains the closure of a nonempty open set.

$D$. For every set $F$ of measure zero and $\varepsilon>0$, there is an open set $G \supset F$ such that $m(G)<\varepsilon$.

Proof. That A and B are equivalent was proven by Schaerf [18] for any topological space. It follows from Theorem 5.1 that the measure $m$ is a category measure for $(X, \mathscr{U})$ and Oxtoby [16] has shown the equivalence of $\mathbf{B}$ and $\mathbf{C}$ for category measures. Finally, we show B is equivalent to $D$. Let $M$ be any measurable set. Suppose D is satisfied. One may write $M=M^{\circ} \cup Z$ where $m(Z)=0$. For $\varepsilon>0$ given, there is an open set $G_{1}$ such that $G_{1} \supset Z$ and $m\left(G_{1}\right)<\varepsilon$. Then if $G=M^{\circ} \cup G_{1}$, we have $m(G) \leqq m\left(M^{\lrcorner}\right)+m\left(G_{1}\right)<m(M)+\varepsilon$. Conversely if B is satisfied then it is obvious that $\mathrm{D}$ is satisfied.

The author does not know whether or not any of the conditions in Theorem 6.1 are satisfied for the general situation we are considering. However, there is a relationship between the measurable functions and continuous functions in our general situation. This result is based on the following theorem due to Levine [13].

6.2. Theorem. A necessary and sufficient condition that a function $f$ be continuous a.e. is that for every open set $W$, the set $f^{-1}(W)$ may be written in the form $f^{-1}(W)=U \cup Z$ where $U$ is open and $m(Z)=0$.

Proof. Sufficiency. Let $E$ be the set of points of discontinuity of $f$ and let $x \in E$. Then there is a neighborhood $W$ of $f(x)$ such that for any open set $U$ which contains $x, U$ is not contained in $f^{-1}(W)$. There is an open interval $N(r, s)$ with rational center $r$ and rational radius such that $f(x) \in N(r, s) \subset W$ and no open set containing $x$ is contained in $f^{-1}(N(r, s))$. Then $f^{-1}(N(r, s))=U_{r, s} \cup Z_{r, s}$ where $U_{r, s}$ is open and $m\left(Z_{r, s}\right)=0$. Since $x \notin U_{r, s}, x \in Z_{r, s}$ and hence $E \subset \bigcup_{r, s} Z_{r, s}$ so that $m(E)=0$.

Necessity. Let $W$ be any open set of real numbers. Then if we write $f^{-1}(W)=C \cup D$, where $C$ denotes the points in $f^{-1}(W)$ where $f$ is continuous and $D$ denotes the points in $f^{-1}(W)$ where $f$ is discontinuous, we have $m(D)=0$. Furthermore for each $x \in C$, there exists an open set $U(x)$ containing $x$ and contained in $f^{-1}(W)$. Thus $f^{-1}(W)=C \cup D \subset \bigcup_{x \in c} U(x) \cup D \subset f^{-1}(W)$ so that $f^{-1}(W)$ may be written as the union of an open set and a set of measure zero.

6.3. Theorem. A necessary and sufficient condition that a function be measurable is that it is continuous almost everywhere.

Proof. Sufficiency. By Theorem 6.2, if $W=\{x: x<c\}, f^{-1}(W)=U \cup Z$ where $U$ is open and $m(Z)=0$. Since open sets are measurable, $f^{-1}(W)$ is a measurable set and $f$ is measurable. 
Necessity. Since $f$ is measurable, $f^{-1}(W)$ is a measurable set for any open set $W$. By Theorem 5.1, $f^{-1}(W)=U \triangle Z$ where $U$ is open and $m(Z)=0$. But $U \triangle Z=(U-Z) \cup(Z-U)$ and since $Z$ is closed, $U-Z$ is open and hence $f^{-1}(W)$ may be written as a union of an open set and a set of measure zero. Therefore by Theorem $6.1, f$ is continuous almost everywhere.

6.4. Corollary. Iff is a function on $X$ to the reals the following are equivalent.

A. $f$ is measurable.

B. $f$ is at most pcintwise discontinuous.

C. $f$ is piecewise continuous, i.e., continuous except for a closed discrete set.

D. $f$ is continuous except for a nowhere dense set.

7. On the first derivative. In this section we shall restrict ourselves to the reals with Lebesgue measure and usual metric density. We shall use a special case of the topology defined in [14] as well as the density topology in order to restate scme theorems concerning the first derivative in topological terms.

First we review a special case of the topology defined in [14]. A point $x$ is called a $\mathscr{W}$-limit point of a set $E$ if every open interval about $x$ intersects $E$ in a set of positive outer measure, i.e., if the set $E$ is metrically dense at $x$. A set will be called $\mathscr{W}$-closed if it contains all its $\mathscr{W}$-limit points. The $\mathscr{W}$-open sets are complements of $\mathscr{W}$-closed sets. It turns out that a necessary and sufficient condition that a set be $\mathscr{W}$-open is that it may be written in the form $G-Z$ where $G$ is a usual open set and $m(Z)=0$. It is clear that if $\mathscr{U}$ is the density topology, then $\mathscr{U}$ is a finer topology than $\mathscr{W}$, and if $\mathscr{T}$ is the usual topology $\mathscr{W}$ is finer than $\mathscr{T}$. It was shown in [14] however that a necessary and sufficient condition that a function to a regular space be $\mathscr{W}$-continuous is that it is $\mathscr{T}$-continuous. The sets of measure zero are characterized as $\mathscr{W}$-closed discrete subsets of the reals.

It is quite easy to show that if $f$ is a function on a space $X$ with no isolated points to a space $Y$ then a necessary and sufficient condition that $f$ be continuous is that for every open set $W \subset Y, f^{-1}(W) \subset \operatorname{der} f^{-1}(W)-\operatorname{der} f^{-1}(Y-W)$, where der denotes the derived set. We shall call a function thick if $f^{-1}(W) \subset \operatorname{der} f^{-1}(W)$ for every open set $W$. Thus in a space with no isolated points a continuous function is thick, but the converse is clearly not true.

7.1. Lemma. A necessary and sufficient condition that a function $f$ on $a$ space $X$ to a space $Y$ be thick is that for every point $x$ in $X$, and every neighborhood $N$ of $f(x), x$ is a limit point of $f^{-1}(N)$.

We will say a function $f$ is thick at a point $x$ if for every open set $N$ containing $f(x), x$ is a limit point of $f^{-1}(N)$.

7.2. TheOREM ( $\left.{ }^{3}\right)$. Let $X$ be a $T_{1}$ space with no isolated points and $Y$ a second

(3) This theorem is the special case of Lemma II of Cargal's paper [4] where one takes the set property $\lambda$ to be that $S$ contains at least two points. 
countable space. Every function on $X$ to $Y$ is thick at each point of the comp ement of a first category set.

Proof. Let $\left\{W_{n}\right\}$ be a countable basis for $Y, f$ be a function on $X$ to $Y$, and let $H$ be the set of points at which $f$ is not thick. For each positive integer $k$ let $N_{k}=f^{-1}\left(W_{k}\right)-\operatorname{der} f^{-1}\left(W_{k}\right)$. Since $X$ is $T_{1}, N_{k}$ is a discrete subset of $X$ and since in a $T_{1}$ space with no isolated points every discrete set is nowhere dense, $N_{k}$ is nowhere dense. If $x$ is any point in $H$, there is a $k$ such that $x \in N_{k}$ and hence $H \subset \bigcup_{k=1}^{\infty} N_{k}$. Since any subset of a first category set is first category, $H$ is a first category set.

A function $f$ will be called a derivative function if it is defined on an interval $X$ (possibly infinite) and there exists a function $F$ differentiable on $X$ and such that $F^{\prime}(x)=f(x)$ for all $x$ in $X$.

\subsection{THEOREM. If $f$ is a derivative function then $f$ is $\mathscr{W}$-thick.}

Proof. Let $x_{0} \in X, \varepsilon>0$, be given, and $I$ be any subinterval of $X$ containing $x_{0}$. From a theorem of Denjoy [8] (see also Clarkson [5]) the set

$$
E=\left\{x:\left|f(x)-f\left(x_{0}\right)\right|<\varepsilon, x \in I\right\}
$$

has positive measure. Since $I$ is arbitrary, $x_{0}$ is a $\mathscr{W}$-limit point of $\left\{x:\left|f(x)-f\left(x_{0}\right)\right|<\varepsilon\right\}$ and $f$ is $\mathscr{W}$-thick at $x_{0}$.

Since $\mathscr{T} \subset \mathscr{W}$ it follows immediately that if $f$ is a derivative function then $f$ is $\mathscr{T}$-thick. It is not true, however, that if $f$ is a derivative function then it is $\mathscr{U}$-thick. In the following example we construct a function $f$ which is a bounded derivative function but has the property that there exists an $\varepsilon>0$ and an $x_{0}$ in the domain of $f$ such that the set

$$
E\left(x_{0}, \varepsilon\right)=\left\{x:\left|f(x)-f\left(x_{0}\right)\right|<\varepsilon\right\}
$$

has density zero at $x_{0}$ and hence $x_{0}$ is not a $\mathscr{U}$-limit point of $E$.

7.4. EXAMPLE. Let $\left\{\lambda_{n}\right\}$ be a strictly monotone increasing sequence of real numbers converging to one. Let

$$
f(x)= \begin{cases}0, & x=0, \\ (-1)^{n+1}, & \frac{1}{n+1}<x<\frac{n+\lambda_{n}}{n(n+1)}, \\ \frac{(-1)^{n}}{1-\lambda_{n}}\left[2 n(n+1) x-\left(2 n+1+\lambda_{n}\right)\right], & \frac{n+\lambda_{n}}{n(n+1)} \leqq x \leqq \frac{1}{n}\end{cases}
$$

for $n=1,2, \cdots$, and extend the domain of $f$ to $[-1,+1]$ by_defining $f(-x)=f(x)$.

Since the function $f$ defined above is bounded and continuous on $[-1,+1]$ except at 0 it is Riemann integrable and we define the function $F(x)=\int_{-1}^{x} f(t) d t$ for $-1 \leqq x \leqq+1$. We shall show that $F^{\prime}(x)=f(x)$ for all $x$ in $[-1,1]$ but $E(0,1)$ has metric density zero at zero. 
It is clear that $F^{\prime}(x)=f(x)$ for $x \neq 0$. Let $h$ be any positive number less than 1 . Then there exists a positive integer $n$ such that $n^{-1} \leqq h<(n-1)^{-1}$ and

$$
\begin{aligned}
\left|\int_{0}^{h} f(t) d t\right| & \leqq\left|\int_{0}^{1 / n} f d t\right|+\int_{1 / n}^{h}|f| d t \\
& \leqq\left|\int_{0}^{1 / n} f d t\right|+\frac{1}{n(n-1)} .
\end{aligned}
$$

From the definition of $f$ we have

$$
\int_{0}^{1 / n} f d t=\sum_{j=n}^{\infty} \frac{(-1)^{j} \lambda_{j}}{j(j+1)}
$$

so that

$$
\left|\int_{0}^{1 / n} f d t\right| \leqq \frac{\lambda_{n}}{n(n+1)}
$$

Therefore

$$
\left|\int_{0}^{h} f d t\right| \leqq \frac{\lambda_{n}}{n(n+1)}+\frac{1}{n(n-1)}
$$

and since $h \geqq 1 / n$ we have

$$
\left|\frac{1}{h} \int_{0}^{h} f d t\right| \leqq \frac{\lambda_{n}}{n+1}+\frac{1}{n-1} .
$$

Since $\lambda_{n}$ converges monotonely to 1 it follows that $\lambda_{n} /(n+1)$ converges to zero and hence the derivative from the right of $F(x)$ at zero is zero. In a similar fashion the derivative from the left at zero is zero and we have $F^{\prime}(0)=f(0)$.

Next we show that the set $E(0,1)=\{x:|f(x)-f(0)|<1\}$ has metric density zero at zero. To this end we examine the complement of $E(0,1)$ in $[-1,1]$, which we denote by $A$, where

$$
A=\bigcup_{n=1}^{\infty}\left[\left\{x: \frac{1}{n+1} \leqq x \leqq \frac{n+\lambda_{n}}{n(n+1)}\right\} \cup\left\{x:-\frac{n+\lambda_{n}}{n(n+1)} \leqq x \leqq-\frac{1}{n+1}\right\}\right] \text {. }
$$

Let $I$ be any interval containing zero of length less than $1 / 2$. Then there exists a positive integer $N$ such that $(N+1)^{-1} \leqq m(I)<N^{-1}$, where $m$ denotes Lebesgue measure. Suppose the right end point of $I$ is between $p^{-1}$ and $(p-1)^{-1}$ and the left end point is between $-(q-1)^{-1}$ and $-q^{-1}$, where $p$ and $q$ are positive integers greater than $N$. Let $J$ be the closed interval $\left[-q^{-1}, p^{-1}\right]$ and denote the complement of $J$ in $I$ by $E$. Since $p, q \geqq N$,

$$
\frac{m(E)}{m(I)} \leqq \frac{2(N+1)}{N(N-1)} .
$$


By the definition of $A$ we have

$$
m(A \cap J)=\sum_{n=p}^{\infty} \frac{\lambda_{n}}{n(n+1)}+\sum_{n=q}^{\infty} \frac{\lambda_{n}}{n(n+1)}>\lambda_{\Lambda} m(J),
$$

since $p, q \geqq N$ and $\lambda_{n}$ is monotone increasing. It follows that

$$
\frac{m(A \cap J)}{m(I)}>\lambda_{N} \frac{m(J)}{m(I)}
$$

Since $m(I)=m(J)+m(E)$, we have

$$
\frac{m(J)}{m(I)}=1-\frac{m(E)}{m(I)} \geqq 1-\frac{2(N+1)}{N(N-1)}
$$

so that

$$
\frac{m(A \cap I)}{m(I)} \geqq \frac{m(A \cap J)}{m(I)}>\lambda_{N}-\frac{2 \lambda_{N}(N+1)}{N(N-1)}
$$

and since $\lambda_{n} \rightarrow 1$ and $\lambda_{N} / N \rightarrow 0$ it follows that the lower metric density of $A$ at zero is one which implies that the metric density of $E(0,1)$ is zero at zero.

A function $f$ is approximately continuous at $x_{0}$ if for each $\varepsilon>0$, the set $\left\{x:\left|f(x)-f\left(x_{0}\right)\right| \geqq \varepsilon\right\}$ has outer density zero at $x_{0}$ (see Saks [17] and Munroe [15]). Thus $f$ is approximately continuous at $x_{0}$ if and only if for every neighborhood $N$ of $f\left(x_{0}\right), x_{0} \notin \operatorname{der}\left(X-f^{-1}(N)\right)$ where der is the $\mathscr{U}$-derived set. Thus a necessary and sufficient condition that a function be approximately continuous at $x_{0}$ is that it is $\mathscr{U}$-continuous at $x_{0}$. From Theorem 6.2 it follows that approximately continuous functions are measurable and measurable functions are approximately continuous almost everywhere. Denjoy [7] has shown that a bounded approximately continuous function is a derivative function. Therefore we have the following theorem.

7.5 THEOREM. Every bounded $\mathscr{U}$-continuous function is a derivativefunction.

The converse of this theorem cannot be true since we have shown that a derivative function need not be even $\mathscr{U}$-thick. One might conjecture that if a function were bounded, $\mathscr{U}$-thick, possessed the Darboux property and was of Baire class I it would be a derivative function. This is not the case as shown by the following example.

7.6. EXAmple. Consider the function $f$ defined by

$$
f(x)= \begin{cases}\sin 1 / x, & x \neq 0, \\ 1, & x=0 .\end{cases}
$$


This function is not a derivative function for the function

$$
g(x)= \begin{cases}\sin 1 / x, & x \neq 0 \\ 0, & x=0\end{cases}
$$

is a derivative function $\left({ }^{4}\right)$, namely the derivative of $\int^{x} g(t) d t$, but the function $f(x)-g(x)$ is not since it does not have the Darboux property.

The function $f$ is a function of Baire class I with the Darboux property, and we will show that the metric density of the set $E(0, \varepsilon)=\{x:|f(x)-f(0)|<\varepsilon\}$ is $\frac{1}{2}-(1 / \pi) \sin ^{-1}(1-\varepsilon)$ for all positive $\varepsilon$ less than 1 .

Let $\varepsilon>0$ be given and suppose $\varepsilon<1$. Let $\delta=1-\varepsilon, A=E(0, \varepsilon)$, $\alpha_{k}=\left[(2 k+1) \pi-\sin ^{-1} \delta\right]^{-1}$, and $\beta_{k}=\left[2 k \pi+\sin ^{-1} \delta\right]^{-1}$. Let $I=(c, d)$ be any open interval containing zero. There exist positive integers $n$ and $m$ such that

$$
-\alpha_{m-1}<c \leqq-\alpha_{m} \text { and } \alpha_{n}<d \leqq \alpha_{n-1}
$$

Then

$$
m(A \cap I) \leqq \sum_{j=n}^{\infty}(\beta j-\alpha j)+\sum_{j=m}^{\infty}(\beta j-\alpha j)
$$

and

$$
m(A \cap I) \geqq \sum_{j=n+1}^{\infty}(\beta j-\alpha j)+\sum_{j=m+1}^{\infty}(\beta j-\alpha j)
$$

and since we have

$$
\alpha_{n}+\alpha_{m}<m(I)<\alpha_{n-1}+\alpha_{m-1}
$$

it follows that

and

$$
\frac{m(A \cap I)}{m(I)} \leqq \frac{1}{\alpha_{n}+\alpha_{m}}\left[\sum_{j=n}^{\infty}(\beta j-\alpha j)+\sum_{j=m}^{\infty}(\beta j-\alpha j)\right]
$$

$$
\frac{m(A \cap I)}{m(I)} \geqq \frac{1}{\alpha_{n-1}+\alpha_{m-1}}\left[\sum_{j=n+1}^{\infty}(\beta j-\alpha j)+\sum_{j=m+1}^{\infty}(\beta j-\alpha j)\right] .
$$

It can be shown that for any $k$

where

$$
\frac{1}{2 \pi} \log \frac{k+a}{k+b} \leqq \sum_{j=k}^{\infty}(\beta j-\alpha j) \leqq \frac{1}{2 \pi} \log \frac{k-1+a}{k-1+b}
$$

(4) The author wishes to thank Professor A. Zygmund for pointing out to him the fact that $\mathbf{g}(\mathbf{x})$ is a derivative function. 


$$
a=\frac{1}{2 \pi}\left[\pi-\sin ^{-1}(1-\varepsilon)\right] \text { and } b=\frac{1}{2 \pi} \sin ^{-1}(1-\varepsilon) .
$$

Therefore we have

$$
\frac{m(A \cap I)}{m(I)} \leqq\left[\frac{1}{n+a}+\frac{1}{m+a}\right]^{-1}\left[\log \frac{n-1+a}{n-1+b}+\log \frac{m-1+a}{m-1+b}\right] .
$$

Suppose $n \geqq m$; then since $\log$ is monotone increasing the left-hand side of the last displayed inequality does not exceed $(n+a) \log (n-1+a) /(n-1+b)$, so that

$$
\frac{m(A \cap I)}{m(I)} \leqq(n+a) \log \frac{n-1+a}{n-1+b} .
$$

In a similar fashion we may show that

$$
\frac{m(A \cap I)}{m(I)} \geqq(m-1+a) \log \frac{m+1+a}{m+1+b} .
$$

Since both $(n+a) \log (n-1+a) /(n-1+b)$ and $(m-1+a) \log (m+1+a) /(m+1+b)$ converge to $a-b$ as $n, m \rightarrow \infty$ we have that the metric density of $A$ at zero is $\frac{1}{2}-(1 / \pi) \sin ^{-1}(1-\varepsilon)$.

Goffman, Neugebauer and Nishiura [10] have shown that the space $(X, \mathscr{U})$ where $X$ is the real line, is completely regular. Thus from Theorem 6.1, Lebesgue measure is a regular Borel measure in the density topology. This would follow however from the D part of 6.1 without the complete regularity. The space however is quite pathological as shown by the following theorem.

7.7. THEOREM. The space $(X, \mathscr{U})$ is Hausdorff, has no nontrivial compact subsets, and is not first countable at any point.

Proof. Since $\mathscr{U}$ is finer than $\mathscr{W}$ and these are properties possessed by $\mathscr{W}$ (see [14]).

\section{REFERENCES}

1. Henry Blumberg, The measurable boundaries of arbitrary functions, Acta Math. 65 (1935), 263-282.

2. - Arbitrary point transformations, Duke Math. J. 11 (1944), 671-685.

3. - New properties of all real functions, Trans. Amer. Math. Soc. 24 (1922), 113-128.

4. Buchannan Cargal, Generalizations of continuity, Proc. Iowa Acad. Sci. 60 (1953), 477-481.

5. J. A. Clarkson, A property of derivatives, Bull. Amer. Math. Soc. 53 (1947), 124-125.

6. W. W. Comfort and Hugh Gordon, Vitali's theorem for invariant measures, Trans. Amer. Math. Soc. 99 (1961), 83-90.

7. A. Denjoy, Sur les fonctions dérivées sommables, Bull. Soc. Math. France 43 (1915), 161-248. 
8. — Sur une propriété des fonctions dérivées, Enseignement Math. 18 (1916), 172.

9. - Une extension du théorème de Vitali, Amer. J. Math. 73 (1951), 314-356.

10. C. Goffman, C. Neugebauer and T. Nishiura, Density topology and approximate continuity, Duke Math. J. 28 (1961), 497-505.

11. C. Goffman and D. Waterman, Approximately continuous transformations, Proc. Amer. Math. Soc. 12 (1961), 116.

12. H. Hahn and A. Rosenthal, Set functions, Univ. of New Mexico Press, Albuquerque, N. M., 1948.

13. Norman Levine, A note on functions continuous almost everywhere, Amer. Math. Monthly 66 (1959), 791-792.

14. N. F. G. Martin, Generalized condensation points, Duke Math. J. 28 (1961), 507-514.

15. M. E. Munroe, Introduction to measure and integration, Addison-Wesley, Cambridge, Mass., 1953.

16. J. C. Oxtoby, Spaces that admit a category measure, J. Reine Angew. Math. 205 (1961), 156-170.

17. S. Saks, Theory of the integral, Hafner, New York, 1937.

18. H. M. Schaerf, On the continuity of measurable functions in neighborhood spaces, Portugal. Math. 6 (1947), 33-44.

19. W. J. Trjitzinsky, Théorie métrique dans les espaces où il y a une mesure, Mémorial des Sciences Mathématiques, Fasc. 143, Gauthier-Villars, Paris, 1960.

\section{UNIVERSITY OF VIRGINIA,}

ChaRlotTesville, Virginia 